\title{
FUTURE TRENDS IN DRUG DELIVERY
}

Dear Reader,

In the ancient times, the Vaidyaraj was expected to develop therapies to prevent or treat diseases. Many times, these physicians prepared medicines freshly using plants cultivated in their gardens or collected from the forests. These were patient based medicines and highly customized for the individual patient (based on nadipariksha or pulse diagnosis or similartechniques). Similar approaches were used in other ancient cultures of the world such as Egypt, Greece and China. Little changed in the method of diagnosis and treatment till the industrial revolution. In the last century, systematic research led to an

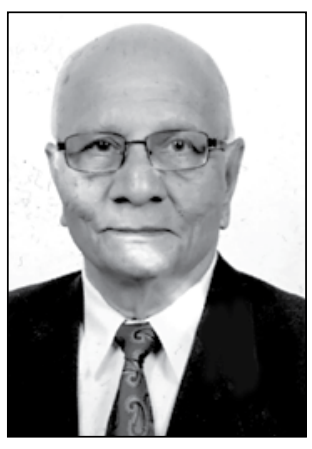
in-depth understanding of cell biology and biochemistry. This paved the path of understanding of cause and progression of disease at the cellular level. This helped the development of synthetic drugs such as analgesics and antibiotics during the Second World War. These drugs had a major advantage over the plant-based medicines as they were specific for the disease through receptor binding and treating the symptoms appropriately.

Although beneficial, some of these medicines have a major disadvantage of their toxicity, sometimes overweighing their effectiveness. Most of the drugs and doses are made for a 'normal' population. However, every person is different from others with respect to age, weight, sex as well as other co-morbidities, which may affect the drug effectiveness as well as the side effect profile. Therefore, there is a need to personalize the medicine in order to make it most effective and safe for everyone. Hence, it is believed that there will be a major shift from generalized to personalized medicine in the future.

Recent advancements in the field of medicine and pharmaceutics have revolutionized pharmaceutical research. While work is still in progress, there is a hope that more research is being conducted to address some of the issues associated with current medicines. The recent advances in drug delivery technology such as nano technology and liposomes, on demand manufacturing techniques such as 3-D printing and biotechnology-based gene and stem-cell therapies headed towards the development of personalized medicines. This will help to improve the effectiveness of the medicines and reduce their overall side effects.

There are several new frontiers in the drug delivery technology which can precisely deliver the medicines to the target tissues and reduce the overall drug exposure to the body. Nanospheres and liposomes are particularly of interest due to their size and the possibility of potential modifications on the surface to target only the morbid tissues and reduce the undesirable side effects. This is predominantly important in oncology, where drugs have severe side effects and drugs can make a difference between life and death. Nano technology continues to evolve with the use of biocompatible particles for the safe and effective delivery of genetic materials.

In terms of technology advancement, 3-D printing of dosage forms offers an exciting platform for customizing medicine. These technologies could produce drug or drug combinations specific to the patients' exact needs, taking into account their weight, age and other health conditions and would facilitate manufacturing the products at the point-of-sale (in pharmacies). This personalized treatment would certainly improve the drug's effectiveness and reduce the side effects. This could also provide unique dosage forms, for example, easy to swallow tablets for seniors and animal shapes for the children. Not every drug can be produced by this technique due to the amount 
of dose, solubility and/or regulatory challenges but it still provides a good option to address some of the issues with the current therapies. Although this technology needs more work, there are already systems under advanced stages of development in the United States.

One of the innovative technologies for the use of personalized medicine is gene therapy. Genes are the blueprints for proteins which serve as building blocks for tissues and regulate the chemical reactions inside the living cells. Gene disorders such as cystic fibrosis, hemophilia and sickle cell anemia are caused by a single gene mutation and are usually inherited. New understanding in biotechnology has helped in understanding the genetic root cause of these inherited diseases. Gene therapy involves removing a precise damaged part of the gene and replacing it with a healthy gene. This requires precisely knowing where and how to replace the mutated gene with a healthy one without altering the rest of the sequence. Due to these challenges, a lot of research is yet to be conducted to formalize this treatment. By getting involved in this research, Indian scientists can provide tremendous value and leadership to this technology.

Another technology being developed for treatment is stem cell based medicines. This up and coming technology uses a person's own stem cells to produce vital growth factors to treat the diseases and repair damaged tissues. These treatments could help to reduce inflammation, fight autoimmune disease, repair and build muscles and joints. Although promising, there are several challenges ahead for this treatment and provides a perfect opportunity for our scientists to contribute to this research.

These are just some of the examples of the latest advancements in drug discovery and development. I hope that many of the young scientists in India will use these techniques and add significant value to the future remedies of this century. I hope their research will pave the road for the personalized therapies to improve patients' quality of life. This also provides an opportunity for Indian scientists to make an impact and become global leaders in healthcare management.

Dr. S. G. Deshpande, Ph.D. (Tech.) Mumbai

\section{About The Guest Editor}

Dr. Sudhakar G. Deshpande is an renowned pharmacist and educator, who served as Principal and Professor of Pharmaceutics at C.U. Shah College of Pharmacy (SNDT University) in Mumbai. Prior to this responsibility, he worked for more than 25 years at the Institute of Chemical Technology (ICT, formerly UDCT) in Mumbai as a Lecturer and Reader in Pharmaceutics. He was honoured as Professor Emeritus and bestowed with a National Professorship by AICTE.

Dr. Deshpande received his B. Sc. (Tech.), M. Sc. (Tech.) and Ph.D. (Tech.) degrees from ICT in Pharmaceutics and continued to conduct research in the area of drug delivery. During his tenure, he guided more than 25 Ph.D. and 100 M. Pharm/M.Sc. (Tech.) students. He co-authored more than 100 research articles in national and international journals and presented his research at several international conferences.

During his professional career of more than 5 decades, he received several recognitions including the prestigious Government of Maharashtra's Best Teachers Award, ML Khorana Memorial Oration Award and Principal of the Year Award. He was selected to represent Indian scientists at PARIS SUD University in France on a sabbatical exchange programme.

Dr. Deshpande served as Chairman, All India Board of Pharmacy Education. He was a member of $\mathrm{PCl}$ and AICTE to accredit pharmacy colleges in India. He served as an advisor to several pharmaceutical companies and institutes in India, US, S. Korea and Canada. Presently he is a consulting editor to IDMA's INDIAN DRUGS journal. 